\title{
A note on degenerate $r$-Stirling numbers
}

Taekyun Kim', Dae San Kim², Hyunseok Lee ${ }^{1}$ and Jin-Woo Park ${ }^{3 *}$ (D)

${ }^{\text {*Correspondence: }}$ a0417001@knu.ac.kr

${ }^{3}$ Department of Mathematics Education, Daegu University, 38453, Daegu, Republic of Korea Full list of author information is available at the end of the article

\begin{abstract}
The aim of this paper is to study the unsigned degenerate $r$-Stirling numbers of the first kind as degenerate versions of the $r$-Stirling numbers of the first kind and the degenerate $r$-Stirling numbers of the second kind as those of the $r$-Stirling numbers of the second kind. For the degenerate $r$-Stirling numbers of both kinds, we derive recurrence relations, generating functions, explicit expressions, and some identities involving them.
\end{abstract}

Keywords: Unsigned degenerate $r$-Stirling numbers of the first kind; Degenerate $r$-Stirling numbers of first kind; Degenerate $r$-Stirling numbers of second kind

\section{Introduction}

Let $[n]=\{1,2,3, \ldots, n\}$. The unsigned Stirling number of the first kind $\left[\begin{array}{l}n \\ k\end{array}\right]$ is the number of permutations of the set $[n]$ with exactly $k$ disjoint cycles, while the Stirling number of the second kind $\left\{\begin{array}{l}n \\ k\end{array}\right\}$ counts the number of partitions of the set $[n]$ into $k$ nonempty disjoint subsets. Let $r$ be a positive integer. The unsigned $r$-Stirling number of the first kind $\left[\begin{array}{l}n \\ k\end{array}\right]_{r}$ is the number of permutations of the set $[n]$ with exactly $k$ disjoint cycles in such a way that the numbers $1,2, \ldots, r$ are in distinct cycles, while the $r$-Stirling number of the second kind $\left\{\begin{array}{l}n \\ k\end{array}\right\}_{r}$ counts the number of partitions of the set $[n]$ into $k$ nonempty disjoint subsets in such a way that the numbers $1,2, \ldots, r$ are in distinct subsets. We remark that Border [1] studied the combinatorial and algebraic properties of the $r$-Stirling numbers.

Carlitz initiated a study of the degenerate Bernoulli and Euler polynomials and numbers. In recent years, some mathematicians have explored various degenerate versions of many special polynomials and numbers by employing various tools like combinatorial methods, generating functions, differential equations, umbral calculus techniques, $p$-adic analysis, and probability theory. These degenerate versions include the degenerate Stirling numbers of the first and second kinds, degenerate Bernoulli numbers of the second kind, and degenerate Bell numbers and polynomials of which interesting arithmetical and combinatorial results were obtained (see [7, 9-11, 13] and references therein). Especially, it turns out that the degenerate Stirling numbers of the first and second kind (see (17), (18)) appear very frequently when we study degenerate versions of some special numbers and polynomials $[7,9,13,16]$.

The aim of this paper is to study the unsigned degenerate $r$-Stirling numbers of the first kind $\left[\begin{array}{l}n \\ k\end{array}\right]_{r, \lambda}$ as degenerate versions of the $r$-Stirling numbers of the first kind $\left[\begin{array}{l}n \\ k\end{array}\right]_{r}$ and the

(c) The Author(s) 2020. This article is licensed under a Creative Commons Attribution 4.0 International License, which permits use, sharing, adaptation, distribution and reproduction in any medium or format, as long as you give appropriate credit to the original author(s) and the source, provide a link to the Creative Commons licence, and indicate if changes were made. The images or other third party material in this article are included in the article's Creative Commons licence, unless indicated otherwise in a credit line to the material. If material is not included in the article's Creative Commons licence and your intended use is not permitted by statutory regulation or exceeds the permitted use, you will need to obtain permission directly from the copyright holder. To view a copy of this licence, visit http://creativecommons.org/licenses/by/4.0/. 
degenerate $r$-Stirling numbers of the second kind $\left\{\begin{array}{l}n \\ k\end{array}\right\}_{r, \lambda}$ as degenerate versions of the $r$ Stirling numbers of the second kind $\left\{\begin{array}{l}n \\ k\end{array}\right\}_{r}$. They can be viewed also as natural extensions of the degenerate Stirling numbers of the first kind $\left[\begin{array}{l}n \\ k\end{array}\right]_{\lambda}$ and the degenerate Stirling numbers of the second kind $\left\{\begin{array}{l}n \\ k\end{array}\right\}_{\lambda}$ which were introduced earlier [7-9, 12]. For the degenerate $r$-Stirling numbers of both kinds, we derive recurrence relations, generating functions, explicit expressions, and some identities involving them.

The outline of this paper is as follows. In Sect. 1, we recall the degenerate exponential functions, the rising and falling $\lambda$-factorial sequences, recurrence relations and generating functions of $r$-Stirling numbers, Lah numbers, the degenerate Stirling numbers and the degenerate logarithm functions. In Sect. 2, we introduce the unsigned degenerate $r$ Stirling numbers of the first kind and the degenerate $r$-Stirling numbers of the second kind and derive recurrence relations, generating functions, expressions of degenerate $r$-Stirling numbers in terms of degenerate Stirling numbers, and a representation of the degenerate $r$-Bell polynomials in terms of the degenerate $r$-Stirling numbers of the second kind.

For any $0 \neq \lambda \in \mathbb{R}$, the degenerate exponential functions are defined by

$$
e_{\lambda}^{x}(t)=(1+\lambda t)^{\frac{x}{\lambda}}=\sum_{n=0}^{\infty}(x)_{n, \lambda} \frac{t^{n}}{n !} \quad(\text { see }[7,10,11])
$$

where the falling $\lambda$-factorial sequence is given by

$$
(x)_{0, \lambda}=1, \quad(x)_{n, \lambda}=x(x-\lambda)(x-2 \lambda) \cdots(x-(n-1) \lambda) \quad(n \geq 1) .
$$

When $x=1$, we have

$$
e_{\lambda}(t)=e_{\lambda}^{1}(t)=\sum_{n=0}^{\infty}(1)_{n, \lambda} \frac{t^{n}}{n !} .
$$

The rising $\lambda$-factorial sequence is defined by

$$
\langle x\rangle_{0, \lambda}=1, \quad\langle x\rangle_{n, \lambda}=x(x+\lambda) \cdots(x+(n-1) \lambda) \quad(n \geq 1) .
$$

When $\lambda=1$, we have

$$
\begin{array}{ll}
(x)_{0}=1, & (x)_{n}=x(x-1) \cdots(x-n+1) \quad(n \geq 1), \\
\langle x\rangle_{0}=1, & \langle x\rangle_{n}=x(x+1) \cdots(x+n-1) \quad(n \geq 1) .
\end{array}
$$

Throughout this paper, we assume that $r$ is any positive integer.

It is known that the $r$-Stirling numbers satisfy the following recurrence relations:

$$
\begin{aligned}
& {\left[\begin{array}{l}
n \\
m
\end{array}\right]_{r}=(n-1)\left[\begin{array}{c}
n-1 \\
m
\end{array}\right]_{r}+\left[\begin{array}{c}
n-1 \\
m-1
\end{array}\right]_{r} \quad(n>r),} \\
& \left\{\begin{array}{l}
n \\
m
\end{array}\right\}_{r}=m\left\{\begin{array}{c}
n-1 \\
m
\end{array}\right\}_{r}+\left\{\begin{array}{c}
n-1 \\
m-1
\end{array}\right\}_{r} \quad(n>r), \\
& {\left[\begin{array}{l}
n \\
m
\end{array}\right]_{r}=0, \quad\left\{\begin{array}{l}
n \\
m
\end{array}\right\}_{r}=0, \quad \text { if } n<r,}
\end{aligned}
$$




$$
\left[\begin{array}{l}
n \\
m
\end{array}\right]_{r}=\delta_{m r}, \quad\left\{\begin{array}{l}
n \\
m
\end{array}\right\}_{r}=\delta_{m r}, \quad \text { if } n=r(\text { see }[1-5,14])
$$

The $r$-Stirling numbers of the first kind have the "horizontal" generating function:

$$
\sum_{k=r}^{n}\left[\begin{array}{l}
n \\
k
\end{array}\right]_{r} z^{k}=z^{r}(z+r) \cdots(z+n-1)=z^{r}\langle z+r\rangle_{n-r}
$$

where $n \geq r$ (see $[1-7,9-11,13,15])$.

Thus, by (10), we get

$$
\langle z+r\rangle_{n}=\sum_{k=0}^{n}\left[\begin{array}{l}
n+r \\
k+r
\end{array}\right]_{r} z^{k} \quad(n \geq 0)
$$

The $r$-Stirling numbers of the second kind have the "vertical" generating function:

$$
\sum_{n=m}^{\infty}\left\{\begin{array}{l}
n \\
m
\end{array}\right\}_{r} z^{n}=\frac{z^{m}}{(1-r z)(1-(r+1) z) \cdots(1-m z)} \quad(m \geq r)
$$

which is equivalent to

$$
\left\{\begin{array}{c}
n+r \\
m+r
\end{array}\right\}_{r}=\frac{1}{m !} \sum_{k=0}^{m}\left(\begin{array}{c}
m \\
k
\end{array}\right)(-1)^{m-k}(r+k)^{n} \quad(n \geq m \geq 0),
$$

and is also equivalent to

$$
\sum_{n=m}^{\infty}\left\{\begin{array}{l}
n+r \\
m+r
\end{array}\right\} \frac{t^{n}}{n !}=\frac{1}{m !}\left(e^{t}-1\right)^{m} e^{r t}
$$

From (14), we note that

$$
(z+r)^{n}=\sum_{k=0}^{n}\left\{\begin{array}{l}
n+r \\
k+r
\end{array}\right\}(z)_{k} \quad(n \geq 0) .
$$

As is well known, the Lah numbers are defined by

$$
L(n, k)=\left(\begin{array}{l}
n-1 \\
k-1
\end{array}\right) \frac{n !}{k !} \quad(n \geq k \geq 0) .
$$

From (16), we note that

$$
\frac{1}{k !}\left(\frac{t}{1-t}\right)^{k}=\sum_{n=k}^{\infty} L(n, k) \frac{t^{n}}{n !} \quad(k \geq 0)(\text { see }[2,4,5]) .
$$

Recently, the degenerate Stirling numbers of the first kind were defined by

$$
(x)_{n}=\sum_{k=0}^{n} S_{1, \lambda}(n, k)(x)_{k, \lambda} \quad(n \geq 0)(\text { see }[1-7,9-11,13,15]),
$$


and the degenerate Stirling numbers of the second kind were defined by

$$
(x)_{n, \lambda}=\sum_{k=0}^{n}\left\{\begin{array}{l}
n \\
k
\end{array}\right\}_{\lambda}(x)_{k} \quad(n \geq 0)(\text { see }[7,9]) .
$$

Note that

$$
\lim _{\lambda \rightarrow 0}\left\{\begin{array}{l}
n \\
k
\end{array}\right\}_{\lambda}=\left\{\begin{array}{l}
n \\
k
\end{array}\right\} \quad(n \geq k \geq 0) .
$$

Let us define the degenerate "unsigned" Stirling numbers of the first kind by

$$
\left[\begin{array}{l}
n \\
k
\end{array}\right]_{\lambda}=(-1)^{n-k} S_{1, \lambda}(n, k) \quad(n \geq k \geq 0)
$$

Then, replacing $x$ by $-x$ in (17), we get

$$
\langle x\rangle_{n}=\sum_{k=0}^{n}\left[\begin{array}{l}
n \\
k
\end{array}\right]_{\lambda}\langle x\rangle_{k, \lambda} \quad(n \geq 0) .
$$

Note that

$$
\lim _{\lambda \rightarrow 0}\left[\begin{array}{l}
n \\
k
\end{array}\right]_{\lambda}=\left[\begin{array}{l}
n \\
k
\end{array}\right]
$$

Let $\log _{\lambda} t$ be the compositional inverse of $e_{\lambda}(t)$, called the degenerate logarithm function. Then we have

$$
\log _{\lambda}(1+t)=\sum_{n=1}^{\infty} \lambda^{n-1}(1)_{n, \frac{1}{\lambda}} \frac{t^{n}}{n !}=\frac{1}{\lambda}\left((1+t)^{\lambda}-1\right) \quad(\text { see [7] })
$$

Note that

$$
e_{\lambda}\left(\log _{\lambda}(1+t)\right)=\log _{\lambda}\left(e_{\lambda}(1+t)\right)=1+t
$$

\section{Degenerate $r$-Stirling numbers}

In view of (11) and (17), we define the unsigned degenerate $r$-Stirling numbers of the first kind by

$$
\langle x+r\rangle_{n}=\sum_{k=0}^{n}\left[\begin{array}{l}
n+r \\
k+r
\end{array}\right]_{r, \lambda}\langle x\rangle_{k, \lambda} \quad(n \geq 0) .
$$

Note from (11) and (22) that

$$
\lim _{\lambda \rightarrow 0}\left[\begin{array}{l}
n+r \\
k+r
\end{array}\right]_{r, \lambda}=\left[\begin{array}{l}
n+r \\
k+r
\end{array}\right]_{r} .
$$


By (22), we get

$$
\begin{aligned}
\sum_{k=0}^{n+1}\left[\begin{array}{c}
n+1+r \\
k+r
\end{array}\right]_{r, \lambda}\langle x\rangle_{k, \lambda} & =\langle x+r\rangle_{n+1} \\
& =\langle x+r\rangle_{n}(x+r+n) \\
& =\sum_{k=0}^{n}\left[\begin{array}{l}
n+r \\
k+r
\end{array}\right]_{r, \lambda}\langle x\rangle_{k, \lambda}(x+k \lambda+r+n-k \lambda) \\
& =\sum_{k=0}^{n}\left[\begin{array}{l}
n+r \\
k+r
\end{array}\right]_{r, \lambda}\langle x\rangle_{k+1, \lambda}+\sum_{k=0}^{n}(n+r-k \lambda)\left[\begin{array}{l}
n+r \\
k+r
\end{array}\right]_{r, \lambda}\langle x\rangle_{k, \lambda} \\
& =\sum_{k=0}^{n+1}\left[\begin{array}{c}
n+r \\
k-1+r
\end{array}\right]_{r, \lambda}\langle x\rangle_{k, \lambda}+\sum_{k=0}^{n}(n+r-k \lambda)\left[\begin{array}{l}
n+r \\
k+r
\end{array}\right]_{r, \lambda}\langle x\rangle_{k, \lambda} \\
& =\sum_{k=0}^{n+1}\left(\left[\begin{array}{c}
n+r \\
k-1+r
\end{array}\right]_{r, \lambda}+(n+r-k \lambda)\left[\begin{array}{l}
n+r \\
k+r
\end{array}\right]_{r, \lambda}\langle x\rangle_{k, \lambda},\right.
\end{aligned}
$$

as

$$
\left[\begin{array}{c}
n+r \\
r-1
\end{array}\right]_{r, \lambda}=0 \text { and }\left[\begin{array}{c}
n+r \\
n+1+r
\end{array}\right]_{r, \lambda}=0
$$

Therefore, we obtain the following theorem.

Theorem 2.1 Let $n, k$ be nonnegative integers. Then we have

$$
\left[\begin{array}{c}
n+1+r \\
k+r
\end{array}\right]_{r, \lambda}=\left[\begin{array}{c}
n+r \\
k-1+r
\end{array}\right]_{r, \lambda}+(n+r-k \lambda)\left[\begin{array}{l}
n+r \\
k+r
\end{array}\right]_{r, \lambda} \quad(n \geq k) .
$$

In particular,

$$
\left[\begin{array}{l}
n \\
k
\end{array}\right]_{r, \lambda}=\left[\begin{array}{l}
n-1 \\
k-1
\end{array}\right]_{r, \lambda}+(n-1-(k-r) \lambda)\left[\begin{array}{c}
n-1 \\
k
\end{array}\right]_{r, \lambda} \quad(n>r, n>k) .
$$

From (22), we note that

$$
\begin{aligned}
\left(\frac{1}{1-t}\right)^{x}\left(\frac{1}{1-t}\right)^{r} & =\sum_{n=0}^{\infty}\langle x+r\rangle_{n} \frac{t^{n}}{n !} \\
& =\sum_{n=0}^{\infty} \sum_{k=0}^{n}\left[\begin{array}{l}
n+r \\
k+r
\end{array}\right]_{r, \lambda}\langle x\rangle_{k, \lambda} \frac{t^{n}}{n !} \\
& =\sum_{k=0}^{\infty}\left(\sum_{n=k}^{\infty}\left[\begin{array}{l}
n+r \\
k+r
\end{array}\right]_{r, \lambda} \frac{t^{n}}{n !}\right)\langle x\rangle_{k, \lambda} .
\end{aligned}
$$


On the other hand, by (21), we get

$$
\begin{aligned}
\left(\frac{1}{1-t}\right)^{x}\left(\frac{1}{1-t}\right)^{r} & =e_{\lambda}^{-x}\left(\log _{\lambda}(1-t)\right)\left(\frac{1}{1-t}\right)^{r} \\
& =\sum_{k=0}^{\infty} \frac{1}{k !}\left(-\log _{\lambda}(1-t)\right)^{k}\left(\frac{1}{1-t}\right)^{r}\langle x\rangle_{k, \lambda}
\end{aligned}
$$

Therefore, by (24) and (25), we obtain the following theorem.

Theorem 2.2 For any integer $k \geq 0$, we have

$$
\frac{1}{k !}\left(-\log _{\lambda}(1-t)\right)^{k}\left(\frac{1}{1-t}\right)^{r}=\sum_{n=k}^{\infty}\left[\begin{array}{l}
n+r \\
k+r
\end{array}\right]_{r, \lambda} \frac{t^{n}}{n !}
$$

From (19) and (20), we have

$$
\frac{1}{k !}\left(-\log _{\lambda}(1-t)\right)^{k}=\sum_{n=k}^{\infty}\left[\begin{array}{l}
n \\
k
\end{array}\right]_{\lambda} \frac{t^{n}}{n !}
$$

Thus, we get

$$
\begin{aligned}
\frac{1}{k !}\left(-\log _{\lambda}(1-t)\right)^{k}\left(\frac{1}{1-t}\right)^{r} & =\left(\sum_{m=k}^{\infty}\left[\begin{array}{c}
m \\
k
\end{array}\right]_{\lambda} \frac{t^{m}}{m !}\right)\left(\sum_{l=0}^{\infty}\langle r\rangle_{l} \frac{t^{l}}{l !}\right) \\
& =\sum_{n=k}^{\infty}\left(\sum_{m=k}^{n}\left(\begin{array}{c}
n \\
m
\end{array}\right)\left[\begin{array}{c}
m \\
k
\end{array}\right]_{\lambda}\langle r\rangle_{n-m}\right) \frac{t^{n}}{n !}
\end{aligned}
$$

Therefore, by Theorem 2.2 and (27), we obtain the following theorem.

Theorem 2.3 For any nonnegative integers $n, k$, with $n \geq k$, we have

$$
\left[\begin{array}{l}
n+r \\
k+r
\end{array}\right]_{r, \lambda}=\sum_{m=k}^{n}\left(\begin{array}{l}
n \\
m
\end{array}\right)\left[\begin{array}{l}
m \\
k
\end{array}\right]_{\lambda}\langle r\rangle_{n-m} .
$$

Now, we observe that

$$
\begin{aligned}
\left(\frac{1}{1-t}\right)^{r} & =\left(1+\frac{t}{1-t}\right)^{r}=\sum_{l=0}^{r}\left(\begin{array}{l}
r \\
l
\end{array}\right)\left(\frac{t}{1-t}\right)^{l}=\sum_{l=0}^{r}(r)_{l} \frac{1}{l !}\left(\frac{t}{1-t}\right)^{l} \\
& =\sum_{l=0}^{r}(r)_{l} \sum_{j=l}^{\infty} L(j, l) \frac{t^{j}}{j !}=\sum_{j=0}^{\infty}\left(\sum_{l=0}^{\min \{r, j\}} L(j, l)(r)_{l}\right) \frac{t^{j}}{j !}
\end{aligned}
$$


By (28), we get

$$
\begin{aligned}
\frac{1}{k !} & \left(-\log _{\lambda}(1-t)\right)^{k}\left(\frac{1}{1-t}\right)^{r} \\
& =\sum_{m=k}^{\infty}\left[\begin{array}{c}
m \\
k
\end{array}\right]_{\lambda} \frac{t^{m}}{m !} \sum_{j=0}^{\infty}\left(\sum_{l=0}^{\min \{r, j\}} L(j, l)(r)_{l}\right) \frac{t^{j}}{j !} \\
& =\sum_{n=k}^{\infty}\left\{\sum_{j=0}^{n-k} \sum_{l=0}^{\min \{r, j\}} L(j, l)(r)_{l}\left(\begin{array}{c}
n \\
j
\end{array}\right)\left[\begin{array}{c}
n-j \\
k
\end{array}\right]_{\lambda}\right\} \frac{t^{n}}{n !} .
\end{aligned}
$$

Therefore, by Theorem 2.2 and (29), we obtain the following theorem.

Theorem 2.4 For any nonnegative integers $n, k$, with $n \geq k$, we have

$$
\left[\begin{array}{l}
n+r \\
k+r
\end{array}\right]_{r, \lambda}=\sum_{j=0}^{n-k} \sum_{l=0}^{\min \{r, j\}} L(j, l)(r)_{l}\left(\begin{array}{l}
n \\
j
\end{array}\right)\left[\begin{array}{c}
n-j \\
k
\end{array}\right]_{\lambda}
$$

By (21), we get

$$
\begin{aligned}
\left(\frac{1}{1-t}\right)^{r+k} & =e_{\lambda}^{-r-k}\left(\log _{\lambda}(1-t)\right) \\
& =\sum_{l=0}^{\infty}\langle r+k\rangle_{l, \lambda} \sum_{n=l}^{\infty}\left[\begin{array}{l}
n \\
l
\end{array}\right]_{\lambda} \frac{t^{n}}{n !} \\
& =\sum_{n=0}^{\infty}\left(\sum_{l=0}^{n}\langle r+k\rangle_{l, \lambda}\left[\begin{array}{l}
n \\
l
\end{array}\right]_{\lambda}\right) \frac{t^{n}}{n !}
\end{aligned}
$$

It is not difficult to show that

$$
\left(\frac{1}{1-t}\right)^{r+k}=\sum_{n=0}^{\infty}\left(\sum_{l=0}^{n}\left[\begin{array}{l}
n+r \\
l+r
\end{array}\right]_{r, \lambda}\langle k\rangle_{l, \lambda}\right) \frac{t^{n}}{n !}
$$

Therefore, by (28), (30), and (31), we obtain the following theorem.

Theorem 2.5 For any nonnegative integers $n, k$, we have

$$
\sum_{l=0}^{\min \{r+k, n\}}(r+k)_{l} L(n, l)=\sum_{l=0}^{n}\langle r+k\rangle_{l, \lambda}\left[\begin{array}{l}
n \\
l
\end{array}\right]_{\lambda}=\sum_{l=0}^{n}\left[\begin{array}{c}
n+r \\
l+r
\end{array}\right]_{r, \lambda}\langle k\rangle_{l, \lambda} .
$$

As an inversion formula of (22), we consider the degenerate $r$-Stirling numbers of second kind given by

$$
(x+r)_{n, \lambda}=\sum_{k=0}^{n}\left\{\begin{array}{l}
n+r \\
k+r
\end{array}\right\}_{r, \lambda}(x)_{k}
$$

where $n \geq 0$ and $r \in \mathbb{N}$. 
Note from (15) and (32) that

$$
\lim _{\lambda \rightarrow 0}\left\{\begin{array}{l}
n+r \\
k+r
\end{array}\right\}_{r, \lambda}=\left\{\begin{array}{l}
n+r \\
k+r
\end{array}\right\}
$$

From (32), we have

$$
\begin{aligned}
& (x+r)_{n+1, \lambda}=(x+r)_{n, \lambda}(x+r-n \lambda) \\
& =\sum_{k=0}^{n}\left\{\begin{array}{l}
n+r \\
k+r
\end{array}\right\}_{r, \lambda}(x)_{k}(x-k+k+r-n \lambda) \\
& =\sum_{k=0}^{n}\left\{\begin{array}{l}
n+r \\
k+r
\end{array}\right\}_{r, \lambda}(x)_{k+1}+\sum_{k=0}^{n}\left\{\begin{array}{l}
n+r \\
k+r
\end{array}\right\}_{r, \lambda}(k+r-n \lambda)(x)_{k} \\
& =\sum_{k=0}^{n+1}\left\{\begin{array}{c}
n+r \\
k-1+r
\end{array}\right\}_{r, \lambda}(x)_{k}+\sum_{k=0}^{n}\left\{\begin{array}{l}
n+r \\
k+r
\end{array}\right\}_{r, \lambda}(k+r-n \lambda)(x)_{k} \\
& =\sum_{k=0}^{n+1}\left(\left\{\begin{array}{c}
n+r \\
k-1+r
\end{array}\right\}_{r, \lambda}+(k+r-n \lambda)\left\{\begin{array}{l}
n+r \\
k+r
\end{array}\right\}_{r, \lambda}\right)(x)_{k},
\end{aligned}
$$

since

$$
\left\{\begin{array}{l}
n+r \\
r-1
\end{array}\right\}_{r, \lambda}=0 \text { and }\left\{\begin{array}{c}
n+r \\
n+1+r
\end{array}\right\}_{r, \lambda}=0 .
$$

Therefore, by (32) and (33), we obtain the following theorem.

Theorem 2.6 For any nonnegative integers $n, k$, with $n \geq k$, we have

$$
\left\{\begin{array}{c}
n+1+r \\
k+r
\end{array}\right\}_{r, \lambda}=\left\{\begin{array}{c}
n+r \\
k-1+r
\end{array}\right\}_{r, \lambda}+(k+r-n \lambda)\left\{\begin{array}{c}
n+r \\
k+r
\end{array}\right\}_{r, \lambda} .
$$

In particular,

$$
\left\{\begin{array}{l}
n \\
k
\end{array}\right\}_{r, \lambda}=\left\{\begin{array}{l}
n-1 \\
k-1
\end{array}\right\}_{r, \lambda}+(k-(n-1-r) \lambda)\left\{\begin{array}{c}
n-1 \\
k
\end{array}\right\}_{r, \lambda},
$$

where $n>r$.

By (32), we get

$$
\begin{aligned}
e_{\lambda}^{x+r}(t) & =\sum_{n=0}^{\infty}(x+r)_{n, \lambda} \frac{t^{n}}{n !}=\sum_{n=0}^{\infty}\left(\sum_{k=0}^{n}\left\{\begin{array}{l}
n+r \\
k+r
\end{array}\right\}_{r, \lambda}(x)_{k}\right) \frac{t^{n}}{n !} \\
& =\sum_{k=0}^{\infty}\left(\sum_{n=k}^{\infty}\left\{\begin{array}{l}
n+r \\
k+r
\end{array}\right\}_{r, \lambda} \frac{t^{n}}{n !}\right)(x)_{k} .
\end{aligned}
$$

On the other hand,

$$
e_{\lambda}^{x+r}(t)=\left(e_{\lambda}(t)-1+1\right)^{x} e_{\lambda}^{r}(t)=\sum_{k=0}^{\infty}\left(\frac{1}{k !}\left(e_{\lambda}(t)-1\right)^{k} e_{\lambda}^{r}(t)\right)(x)_{k} .
$$

Therefore, by (34) and (35), we obtain the following theorem. 
Theorem 2.7 For any nonnegative integer $k$, we have

$$
\frac{1}{k !}\left(e_{\lambda}(t)-1\right)^{k} e_{\lambda}^{r}(t)=\sum_{n=k}^{\infty}\left\{\begin{array}{l}
n+r \\
k+r
\end{array}\right\}_{r, \lambda} \frac{t^{n}}{n !}
$$

Now, we observe that

$$
\begin{aligned}
\frac{1}{k !}\left(e_{\lambda}(t)-1\right)^{k} e_{\lambda}^{r}(t) & =\frac{1}{k !} \sum_{j=0}^{k}\left(\begin{array}{l}
k \\
j
\end{array}\right)(-1)^{k-j} e_{\lambda}^{j+r}(t) \\
& =\frac{1}{k !} \sum_{j=0}^{k}\left(\begin{array}{l}
k \\
j
\end{array}\right)(-1)^{k-j} \sum_{n=0}^{\infty}(j+r)_{n, \lambda} \frac{t^{n}}{n !} \\
& =\sum_{n=0}^{\infty}\left\{\frac{1}{k !} \sum_{j=0}^{k}\left(\begin{array}{l}
k \\
j
\end{array}\right)(-1)^{k-j}(j+r)_{n, \lambda}\right\} \frac{t^{n}}{n !} .
\end{aligned}
$$

Therefore, by Theorem 2.7 and (36), we obtain the following theorem.

Theorem 2.8 For any nonnegative integers $n, k$, we have

$$
\frac{1}{k !} \sum_{j=0}^{k}\left(\begin{array}{l}
k \\
j
\end{array}\right)(-1)^{k-j}(j+r)_{n, \lambda}= \begin{cases}\left\{\begin{array}{l}
n+r \\
k+r
\end{array}\right\}_{r, \lambda}, & \text { if } n \geq k \\
0, & \text { otherwise. }\end{cases}
$$

In particular,

$$
\frac{1}{(k-r) !} \sum_{j=0}^{k-r}\left(\begin{array}{c}
k-r \\
j
\end{array}\right)(-1)^{k-r-j}(j+r)_{n-r, \lambda}=\left\{\begin{array}{l}
n \\
k
\end{array}\right\}_{r, \lambda} \quad(n \geq k \geq r)
$$

The double generating function is given by

$$
\begin{aligned}
e_{\lambda}\left(t\left(e_{\lambda}(x)-1\right)\right) e_{\lambda}^{r}(x) & =\sum_{m=0}^{\infty} t^{m}(1)_{m, \lambda} \frac{1}{m !}\left(e_{\lambda}(x)-1\right)^{m} e_{\lambda}^{r}(x) \\
& =\sum_{m=0}^{\infty} t^{m}(1)_{m, \lambda} \sum_{k=m}^{\infty}\left\{\begin{array}{l}
k+r \\
m+r
\end{array}\right\}_{r, \lambda} \frac{x^{k}}{k !} \\
& =\sum_{k=0}^{\infty}\left(\sum_{m=0}^{k} t^{m}(1)_{m, \lambda}\left\{\begin{array}{l}
k+r \\
m+r
\end{array}\right\}_{r, \lambda}\right) \frac{x^{k}}{k !} .
\end{aligned}
$$

Let us define the degenerate $r$-Bell polynomials as follows:

$$
e_{\lambda}\left(t\left(e_{\lambda}(x)-1\right)\right) e_{\lambda}^{r}(x)=\sum_{n=0}^{\infty} \operatorname{Bel}_{n, \lambda}^{(r)}(t) \frac{x^{n}}{n !}
$$

From (37) and (38), we have

$$
\operatorname{Bel}_{n, \lambda}^{(r)}(t)=\sum_{m=0}^{n} t^{m}(1)_{m, \lambda}\left\{\begin{array}{l}
n+r \\
m+r
\end{array}\right\}_{r, \lambda} \quad(n \geq 0) .
$$


Theorem 2.9 For any nonnegative integer $n$, we have

$$
\operatorname{Bel}_{n, \lambda}^{(r)}(t)=\sum_{m=0}^{n}\left\{\begin{array}{l}
n+r \\
m+r
\end{array}\right\}_{r, \lambda}(1)_{m, \lambda} t^{m}
$$

By Theorem 2.7, we get

$$
\begin{aligned}
& \frac{1}{k !}\left(e_{\lambda}(t)-1\right)^{k} e_{\lambda}^{r}(t)=\frac{1}{k !}\left(e_{\lambda}(t)-1\right)^{k}\left(e_{\lambda}(t)-1+1\right)^{r} \\
& =\sum_{j=0}^{r}\left(\begin{array}{l}
r \\
j
\end{array}\right) \frac{1}{k !}\left(e_{\lambda}(t)-1\right)^{k+j} \\
& =\sum_{j=0}^{r}(r)_{j}\left(\begin{array}{c}
k+j \\
k
\end{array}\right) \sum_{n=j+k}^{\infty}\left\{\begin{array}{c}
n \\
j+k
\end{array}\right\} \frac{t^{n}}{n !} \\
& =\sum_{n=k}^{\infty}\left\{\sum_{j=0}^{\min \{r, n-k\}}(r)_{j}\left(\begin{array}{c}
k+j \\
k
\end{array}\right)\left\{\begin{array}{c}
n \\
j+k
\end{array}\right\}_{\lambda}\right\} \frac{t^{n}}{n !} .
\end{aligned}
$$

From (39), we have

$$
\left\{\begin{array}{l}
n+r \\
k+r
\end{array}\right\}_{r, \lambda}=\sum_{j=0}^{\min \{r, n-k\}}(r)_{j}\left(\begin{array}{c}
k+j \\
k
\end{array}\right)\left\{\begin{array}{c}
n \\
j+k
\end{array}\right\}_{\lambda}
$$

Therefore, by (40), we obtain the following theorem.

Theorem 2.10 For any nonnegative integers $n, k$, with $n \geq k$, we have

$$
\left\{\begin{array}{l}
n+r \\
k+r
\end{array}\right\}_{r, \lambda}=\sum_{j=0}^{\min \{r, n-k\}}(r)_{j}\left(\begin{array}{c}
k+j \\
k
\end{array}\right)\left\{\begin{array}{c}
n \\
j+k
\end{array}\right\}_{\lambda} .
$$

Now, we observe that

$$
\begin{aligned}
\frac{1}{k !}\left(-\log _{\lambda}(1-t)\right)^{k}\left(\frac{1}{1-t}\right)^{r} & =\frac{1}{k !}\left(-\log _{\lambda}(1-t)\right)^{k} e_{\lambda}^{-r}\left(\log _{\lambda}(1-t)\right) \\
& =\frac{1}{k !}\left(-\log _{\lambda}(1-t)\right)^{k} \sum_{l=0}^{\infty}\langle r\rangle_{l, \lambda} \frac{1}{l !}\left(-\log _{\lambda}(1-t)\right)^{l} \\
& =\sum_{l=0}^{\infty} \frac{(k+l) !}{k ! l !}\langle r\rangle_{l, \lambda} \frac{1}{(k+l) !}\left(-\log _{\lambda}(1-t)\right)^{k+l} \\
& =\sum_{l=0}^{\infty}\left(\begin{array}{c}
k+l \\
l
\end{array}\right)\langle r\rangle_{l, \lambda} \sum_{n=k+l}^{\infty}\left[\begin{array}{c}
n \\
k+l
\end{array}\right]_{\lambda} \frac{t^{n}}{n !} \\
& =\sum_{n=k}^{\infty}\left(\sum_{l=0}^{n-k}\left(\begin{array}{c}
k+l \\
l
\end{array}\right)\langle r\rangle_{l, \lambda}\left[\begin{array}{c}
n \\
k+l
\end{array}\right]_{\lambda}\right) \frac{t^{n}}{n !}
\end{aligned}
$$


By Theorem 2.2 and (41), we get

$$
\left[\begin{array}{c}
n+r \\
k+r
\end{array}\right]_{r, \lambda}=\sum_{l=0}^{n-k}\left(\begin{array}{c}
k+l \\
l
\end{array}\right)\left[\begin{array}{c}
n \\
k+l
\end{array}\right]_{\lambda}\langle r\rangle_{l, \lambda},
$$

where $n, k$ are nonnegative integers, with $n \geq k$.

Therefore, by (42), we obtain the following theorem.

Theorem 2.11 For any nonnegative integers $n, k$, with $n \geq k$, we have

$$
\left[\begin{array}{c}
n+r \\
k+r
\end{array}\right]_{r, \lambda}=\sum_{l=0}^{n-k}\left(\begin{array}{c}
k+l \\
l
\end{array}\right)\left[\begin{array}{c}
n \\
k+l
\end{array}\right]_{\lambda}\langle r\rangle_{l, \lambda} .
$$

\section{Conclusion}

In this paper, we studied the unsigned degenerate $r$-Stirling numbers of the first kind $\left[\begin{array}{l}n \\ k\end{array}\right]_{r, \lambda}$ as degenerate versions of the $r$-Stirling numbers of the first kind $\left[\begin{array}{l}n \\ k\end{array}\right]_{r}$ and the degenerate $r$ Stirling numbers of the second kind $\left\{\begin{array}{l}n \\ k\end{array}\right\}_{r, \lambda}$ as degenerate versions of the $r$-Stirling numbers of the second kind $\left\{\begin{array}{l}n \\ k\end{array}\right\}_{r}$. They can be viewed also as natural extensions of the degenerate Stirling numbers of the first kind $\left[\begin{array}{l}n \\ k\end{array}\right]_{\lambda}$ and the degenerate Stirling numbers of the second kind $\left\{\begin{array}{l}n \\ k\end{array}\right\}_{\lambda}$, which were introduced earlier. For the degenerate $r$-Stirling numbers of both kinds, we derived recurrence relations, generating functions, expressions of degenerate $r$-Stirling numbers in terms of degenerate Stirling numbers, and a representation of the degenerate $r$-Bell polynomials in terms of the degenerate $r$-Stirling numbers of the second kind.

As it turns out, the degenerate Stirling numbers appear very frequently when we study degenerate versions of many special numbers and polynomials $[7,9,13]$. It would be very interesting to discover many appearances of the degenerate $r$-Stirling numbers in such studies. It is one of our future projects to continue to explore degenerate versions of some special numbers and polynomials and their applications not only in mathematics but also in other disciplines like statistics, physics, engineering, and social sciences.

Acknowledgements

Authors are thankful to the referees for their useful suggestions.

Funding

This research was supported by the Daegu University Research Grant, 2020.

Availability of data and materials

Not applicable.

Competing interests

The authors declare no conflict of interest.

Authors' contributions

TK and DSK conceived of the framework and structured the whole paper; DSK and TK wrote the paper; HL and JWP checked the results of the paper and typed the paper; DSK and TK completed the revision of the article. All authors have read and agreed to the final version of the manuscript. All authors read and approved the final manuscript.

'Department of Mathematics, Kwangwoon University, 139-701, Seoul, Republic of Korea. ${ }^{2}$ Department of Mathematics, Sogang University, 121-742, Seoul, Republic of Korea. ${ }^{3}$ Department of Mathematics Education, Daegu University, 38453, Daegu, Republic of Korea. 


\section{Publisher's Note}

Springer Nature remains neutral with regard to jurisdictional claims in published maps and institutional affiliations.

Received: 24 July 2020 Accepted: 17 September 2020 Published online: 23 September 2020

\section{References}

1. Broder, A.: The $r$-Stirling numbers. Discrete Math. 49(3), 241-259 (1984)

2. Carlitz, L.: Weighted Stirling numbers of the first and second kind. I. Fibonacci Q. 18(2), 147-162 (1980)

3. Carlitz, L.: Weighted Stirling numbers of the first and second kind. II. Fibonacci Q. 18(3), 242-257 (1980)

4. Comtet, L.: Analyse combinatoire, Tomes I, II (French) Collection SUP: "Le Mathématicien", 4, 5. Presses Universitaires de France, Paris (1970)

5. Comtet, L.: Advanced Combinatorics: The Art of Finite and Infinite Expansions, Revised and enlarged edn. Reidel, Dordrecht (1974)

6. Kim, D.S., Kim, T.: A note on polyexponential and unipoly functions. Russ. J. Math. Phys. 26(1), 40-49 (2019)

7. Kim, D.S., Kim, T.: A note on a new type of degenerate Bernoulli numbers. Russ. J. Math. Phys. 27(2), $227-235$ (2020)

8. Kim, D.S., Kim, T.: Degenerate Sheffer sequences and $\lambda$-Sheffer sequences. J. Math. Anal. Appl., 124521 (2020). https://www.sciencedirect.com/science/article/pii/S0022247X20306831

9. Kim, T.: A note on degenerate Stirling polynomials of the second kind. Proc. Jangjeon Math. Soc. 20(3), 319-331 (2017)

10. Kim, T., Kim, D.S.: Degenerate Laplace transform and degenerate gamma function. Russ. J. Math. Phys. 24(2), 241-248 (2017)

11. Kim, T., Kim, D.S.: Degenerate polyexponential functions and degenerate Bell polynomials. J. Math. Anal. Appl. 487(2), $124017(2020)$

12. Kim, T., Kim, D.S., Kim, H.-Y., Lee, H., Jang, L.-C.: Degenerate poly-Bernoulli polynomials arising from degenerate polylogarithm. Adv. Differ. Equ. 2020, Article ID 444 (2020)

13. Kim, T., Yao, Y., Kim, D.S., Jang, G.-W.: Degenerate $r$-Stirling numbers and r-Bell polynomials. Russ. J. Math. Phys. 25(1), 44-58 (2018)

14. Ma, Y., Kim, D. S., Kim, T., Lee, H.: Some identities of Lah-Bell polynomials. Adv. Differ. Equ. 2020, Article ID 510 (2020)

15. Riordan, J.: Combinatorial Identities. Wiley, New York (1968)

16. Simsek, Y:: Generating functions for generalized Stirling type numbers, array type polynomials, Eulerian type polynomials and their applications. Fixed Point Theory Appl. 2013, Article ID 87 (2013)

\section{Submit your manuscript to a SpringerOpen ${ }^{\circ}$ journal and benefit from:}

- Convenient online submission

- Rigorous peer review

- Open access: articles freely available online

- High visibility within the field

- Retaining the copyright to your article

Submit your next manuscript at $\gg$ springeropen.com 